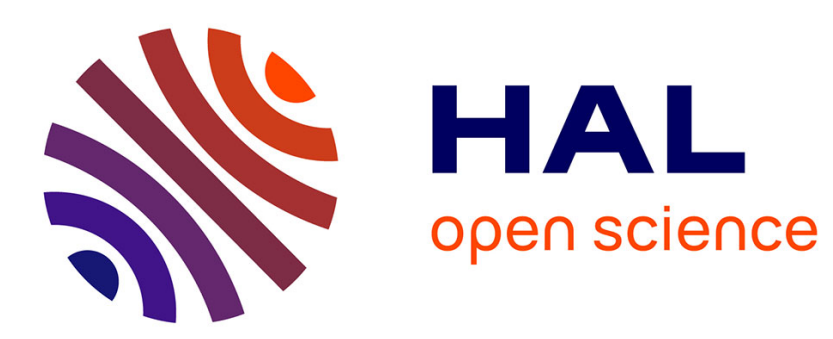

\title{
Singularity and workspace analysis of 3-SPS-U and 4-SPS-U tensegrity mechanisms
}

\author{
Swaminath Venkateswaran, Damien Chablat
}

\section{To cite this version:}

Swaminath Venkateswaran, Damien Chablat. Singularity and workspace analysis of 3-SPS-U and 4-SPS-U tensegrity mechanisms. Advances in Robot Kinematics 2020, 15, 2020, 10.1007/978-3-03050975-0_28. hal-02568290

\section{HAL Id: hal-02568290 \\ https://hal.science/hal-02568290}

Submitted on 8 May 2020

HAL is a multi-disciplinary open access archive for the deposit and dissemination of scientific research documents, whether they are published or not. The documents may come from teaching and research institutions in France or abroad, or from public or private research centers.
L'archive ouverte pluridisciplinaire HAL, est destinée au dépôt et à la diffusion de documents scientifiques de niveau recherche, publiés ou non, émanant des établissements d'enseignement et de recherche français ou étrangers, des laboratoires publics ou privés. 


\title{
Singularity and workspace analysis of 3-SPS-U and 4-SPS-U tensegrity mechanisms
}

\author{
Swaminath Venkateswaran ${ }^{1}$ and Damien Chablat ${ }^{2}$
}

\begin{abstract}
This article analyzes the singularities and workspace of two tensegrity mechanisms that employ a passive universal joint and either three or four tension springs. These two architectures are correlated to 3-SPS-U and 4-SPS-U parallel mechanisms for determining their geometric equations. By fixing the limits of prismatic joints, the workspace for the mechanisms is generated and the parallel singularities are analyzed. Based on the singularity boundaries obtained from the workspace, the joint limits are modified to generate the maximal singularity free workspaces for both the architectures. A comparison is done based on the tilt limits obtained from the workspace of the mechanisms. The mechanism with the maximum tilt limits is implemented for a piping inspection robot to pass through pipe bends and junctions.
\end{abstract}

Key words: Tensegrity mechanism, Singularities, Workspace analysis, 3-SPS-U, 4-SPS-U, Piping inspection robot

\section{Introduction}

A bio-inspired piping inspection robot was designed and developed at LS2N, France. This robot accomplishes the locomotion of a caterpillar in six steps to move inside a pipeline [1]. However, the prototype is a rigid model which restricts its application to straight pipelines. By the addition of articulation units, the robot can be made reconfigurable. Some interesting researches on piping inspection robots that pass through pipe bends include i) the robot of Chen et al. [2] where double hook joint is employed, ii) THES-I robot of Hirose et al. [3] where a universal joint actuated by DC-motor is used and iii) robot of Brunete et al. [4] that uses SMA spring with microcontrollers. Most of these researches have articulation units either in passive mode or active mode but not a combination of both. By analyzing key design issues namely passive compliance, active compliance and tilt limits, a tensegrity mechanism that uses a passive universal joint and three tension springs was proposed as a solution for the bio-inspired robot of LS2N [5]. This mechanism can work passively when the robot passes through a pipe bend at $\pi / 2$ radi-

\footnotetext{
${ }^{1}$ École Centrale de Nantes, Laboratoire des Sciences du Numérique de Nantes (LS2N), UMR CNRS 6004, Nantes, 44300, France, e-mail: swaminath.venkateswaran@1s2n.fr

${ }^{2}$ Centre National de la Recherche Scientifique (CNRS),Laboratoire des Sciences du Numérique de Nantes (LS2N), UMR CNRS 6004, Nantes, 44300, France, e-mail: damien.chablat@cnrs.fr
} 
ans. In the event of a T-union or junction, cable actuation can be performed on the tensegrity mechanism to follow a given path. In this article, the singularities and workspace analysis of the tensegrity mechanism is carried out for the Euler angles of the universal joint [6]. This analysis can help us to determine if the mechanism can work within the singularity boundaries under passive modes. Two types of tensegrity mechanisms are studied and compared where one configuration uses three tensions springs and the other uses four tensions springs. By using the SIROPA library in Maple, the workspace is generated by setting joint limits and parallel singularities. By analyzing these boundaries, the joint limits are modified to obtain singularity free workspaces. By comparing the tilt limits of both the architectures, the mechanism with maximum tilt range is implemented for the bio-inspired robot to overcome the issue of passive compliance.

The outline of the article is as follows. In the following section, the architectures and the geometric constraints of both the mechanisms are presented. The subsequent section deals with the derivation of singularities and joint limits equations. Followed by that, the workspace of the mechanisms are studied and a comparison is being made based on the tilt limits. The article then ends with conclusions.

\section{Architecture of the mechanisms}

The tensegrity mechanisms under study uses a passive universal joint and three tensions springs for one configuration and four springs in the other. These mechanisms can be correlated to parallel manipulators of type 3-SPS-U [7] and 4-SPS-U [8]. Here, $\mathrm{S}$ indicates the spherical joints, $\mathrm{U}$ represents the universal joint and $\underline{\mathrm{P}}$ represents the actuated prismatic joints. The architectures are represented in Figure 1 in the home pose where the tilt angles $\alpha \& \beta$ are equal to 0 radians.
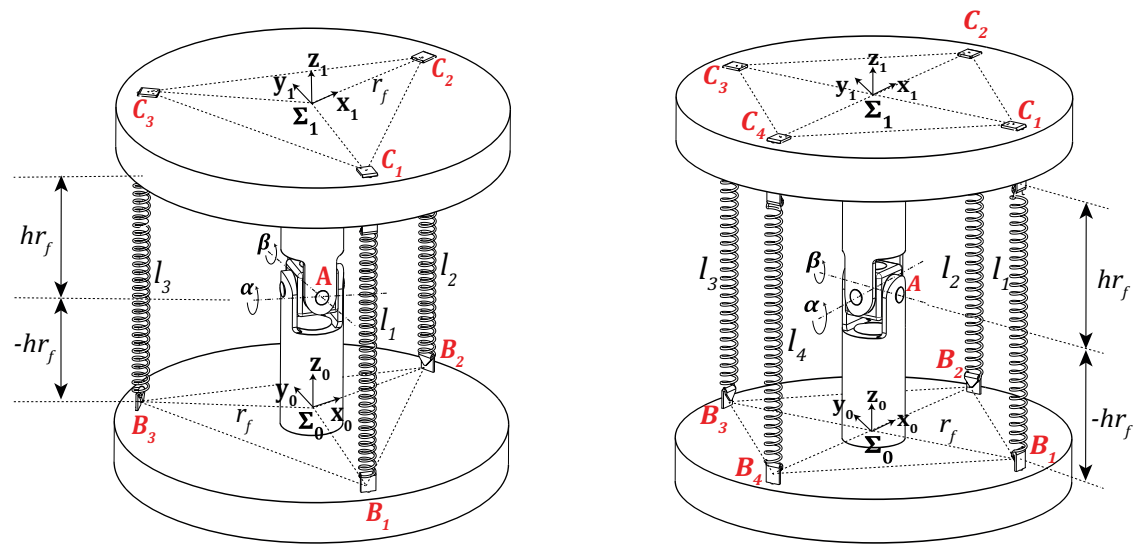

Fig. 1 The 3-SPS-U(left) and 4-SPS-U(right) tensegrity mechanisms at their home poses 
The fixed co-ordinate frame of the base is represented by $\sum_{0}$ with the origin at $B_{0}$. The spring mounting points on the fixed base are represented by $B_{i}(i=1,2,3)$ and they form the imaginary equilateral triangle of the manipulator base whose median is $r_{f}$ for the 3-SPS-U mechanism. The base mouting points $B_{i}(i=1,2,3,4)$ forms an imaginary square for the 4-SPS-U mechanism. The diagonal length for the square is $2 r_{f}$. The vector co-ordinates for the base mounting points are given by

$$
\mathbf{b}_{i}=\left[r_{f} \cos \left(\frac{i 2 \pi}{j}\right), r_{f} \sin \left(\frac{i 2 \pi}{j}\right),-r_{f} h\right]^{T}
$$

where for $j=3, i=0$ to 2 for 3 -S $\underline{P S}-\mathrm{U} \&$ for $j=4, i=0$ to 3 for 4-SPS-U

In Equation 1, $h$ represents a constant and a value of 1 is taken which corresponds to the inverse pendulum configuration [5]. The moving co-ordinate frame of the endeffector is represented by $\Sigma_{1}$ with its origin at $C_{0}$. The spring mounting points of the end-effector is represented by $C_{i}(i=1,2,3)$ and $C_{i}(i=1,2,3,4)$ for the 3-SPS-U and 4-SPS-U mechanisms. For estimating the vector co-ordinates of the end-effector mounting points, the $X Y$ Euler angle matrix is employed with respect to the central point $A$ of the universal joint. The vector co-ordinates for the end-effector mounting points are given by

$$
\begin{gathered}
\mathbf{R}=\mathbf{R}_{x}(\alpha) \mathbf{R}_{y}(\beta)=\left[\begin{array}{ccc}
c_{\beta} & 0 & s_{\beta} \\
s_{\alpha} s_{\beta} & c_{\alpha} & -s_{\alpha} c_{\beta} \\
-c_{\alpha} s_{\beta} & s_{\alpha} & c_{\alpha} c_{\beta}
\end{array}\right] \\
\mathbf{c}_{i}=\mathbf{R}\left[r_{f} \cos \left(\frac{i 2 \pi}{j}\right), r_{f} \sin \left(\frac{i 2 \pi}{j}\right), r_{f} h\right]^{T}
\end{gathered}
$$

where for $j=3, i=0$ to 2 for 3 -S $\underline{P S}-\mathrm{U} \&$ for $j=4, i=0$ to 3 for 4-SP्PS-U

In Equation 2, $\mathbf{R} \in S E(3)$ represents the spatial transformation matrix obtained from the Euler angles of universal joint and is used to identify the end-effector co-ordinates as indicated in Equation 3. The inverse geometric model for the mechanism is simpler and it is used to determine the length of springs between the base and end-effector at home-pose and working conditions. The equation is given by

$$
l_{i}=\sqrt{\left(b_{i x}-c_{i x}\right)^{2}+\left(b_{i y}-c_{i y}\right)^{2}+\left(b_{i z}-c_{i z}\right)^{2}}
$$

with $i=1$ to 3 for 3 -S $\underline{P S}-\mathrm{U} \& i=1$ to 4 for 4 -S $\underline{P S}-\mathrm{U}$

\section{Analysis of the mechanisms}

\subsection{Singularities and joint limits equations}

For determining the feasible workspace of the tensegrity mechanism, it is necessary to determine the singularities of the mechanism. For a parallel manipulator, the 
singularity equation is given by the well-known equation [9]

$$
\mathbf{A t}+\mathbf{B} \dot{\rho}=0
$$

where $\mathbf{t}$ represents the angular velocity vector and $\dot{\rho}=\left[\dot{l}_{1}, \dot{l_{2}}, \dot{l}_{3}\right]^{T}$ represents the joint velocity vector

Parallel singularities occur when the determinant of the direct kinematics matrix A of Equation 5 vanishes. This occurs when the end-effector platform aligns itself with one of the springs of the mechanism. There exist no serial singularities for the mechanism as the determinant of the inverse kinematics matrix $\mathbf{B}$ does not vanish since the prismatic springs cannot be equal to $0 \mathrm{~mm}$. The parallel singularities are analyzed by sub-dividing the manipulator into three and four sets of 2-SPS$\mathrm{U}$ architecture for the 3-SPS-U and 4-SPS-U mechanisms. By using the Groebner base elimination technique, we can generate the joint limits equations with the help of the SIROPA library [10] in Maple. These equations can be generated using the ConstraintEquations syntax of the SIROPA library. The joint limit equations for the 3-SPS-U mechanism are given by

$$
\begin{gathered}
\mathscr{C}_{1+3 i}: 2 r_{f}^{2} c_{\alpha}\left(c_{\beta}-s_{\beta}\right)-2 r_{f}^{2}\left(s_{\beta}+c_{\beta}\right)+4 r_{f}^{2}-l_{j}^{2}=0 \\
\mathscr{C}_{2+(k-1)+3 i}:-m\left(\left(c_{\beta}+\frac{s_{\beta}}{2}+1\right) s_{\alpha} r f^{2} \sqrt{3}+2\left(c_{\beta}+\frac{s_{\beta}}{2}-\frac{3}{4}\right) r f^{2} c_{\alpha}+\right. \\
\left.r_{f}^{2}\left(s_{\beta}-\frac{c_{\beta}}{2}+4\right)-l_{j}^{2}\right)=0
\end{gathered}
$$

for $i=[0,1], l_{j}=\left[l_{\min }, l_{\max }\right] \&$ for $k=[1,2], m=[-1,1]$ in Equation 7

Similarly, the joint limit equations are generated for the 4-SPS-U mechanism and they are given by

$$
\begin{aligned}
& \mathscr{C}_{1+2 k+4 i}: 2 r_{f}^{2} c_{\beta}\left(c_{\alpha}-1\right)+m 2 r_{f}^{2} s_{\beta}\left(c_{\alpha}+1\right)+4 r_{f}^{2}-l_{j}^{2}=0 \\
& \mathscr{C}_{2+2 k+4 i}: 2 r_{f}^{2} c_{\alpha}\left(c_{\beta}-1\right)-m 2 r_{f}^{2} s_{\alpha}\left(c_{\beta}+1\right)+4 r_{f}^{2}-l_{j}^{2}=0
\end{aligned}
$$

for $i=[0,1], l_{j}=\left[l_{\min }, l_{\max }\right] \&$ for $k=[0,1], m=[-1,1]$ in Equations $8 \& 9$

The singularity equations are generated for both the mechanisms to determine their workspace limits. The equations can be generated using ParallelSingularities syntax of the SIROPA library in Maple. Equations 10 to 11 and Equations 12 to 13 represents the singularity equations for the 3-SPS-U and 4-SPS-U mechanisms. These joint limits and singularity equations are employed to determine the singularity free workspace for the mechanisms.

By using the Cylindrical Algebraic Decomposition (CAD) technique, the real solutions are generated for the problem [11] by using the CellDecompositionPlus syntax of the SIROPA library. For isolating the aspects around home-pose, we transform the singularity and joint limits equations as inequalities [12] in Maple. 
Singularity equations for the 3-SPS-U mechanism

$$
\begin{aligned}
& \zeta_{1}: 4 s_{\beta} c_{\beta} c_{\alpha}-14 s_{\beta} c_{\alpha}^{2}+3 c_{\beta}^{2} c_{\alpha}+7 c_{\beta} c_{\alpha}^{2}-6 s_{\beta} c_{\beta}+2 c_{\alpha} s_{\beta}+8 c_{\beta}^{2}+4 c_{\alpha} c_{\beta} \\
& +6 s_{\beta}-3 c_{\beta}+c_{\alpha}-4=0 \\
& \zeta_{1+k}:\left(\left(2 c_{\beta}+2 s_{\beta}+3\right) c_{\alpha}^{2}+\left(3 c_{\beta}^{2}+\left(2-s_{\beta}\right) c_{\beta}-2 s_{\beta}-1\right) c_{\alpha}+c_{\beta}^{2}+3 c_{\beta} s_{\beta}\right. \\
& -2) \sqrt{3}+m\left(3 s_{\alpha}\left(\left(2-s_{\beta}-c_{\beta}\right) c_{\alpha}+3 c_{\beta}^{2}-\left(s_{\beta}+1\right) c_{\beta}+s_{\beta}-1\right)\right)=0
\end{aligned}
$$

for $k=[1,2], m=[-1,1]$ in Equation 11

Singularity equations for the 4-SPS-U mechanism

$$
\begin{aligned}
& \zeta_{1+2 i}: s_{\alpha} s_{\beta} c_{\beta}+s_{\alpha} s_{\beta} c_{\alpha}+j\left(s_{\alpha} c_{\beta}^{2}-s_{\alpha} c_{\beta} c_{\alpha}+s_{\beta} c_{\beta} c_{\alpha}-s_{\beta} c_{\alpha}^{2}-s_{\alpha} c_{\beta}+s_{\alpha} c_{\alpha}\right. \\
& \left.-s_{\beta} c_{\beta}+c_{\alpha} s_{\beta}\right)+c_{\beta}^{2} c_{\alpha}+c_{\beta} c_{\alpha}^{2}-s_{\alpha} s_{\beta}+c_{\beta}^{2}+c_{\alpha} c_{\beta}+c_{\alpha}^{2}-1=0 \\
& \zeta_{2+2 i}: j\left(s_{\alpha} s_{\beta} c_{\beta}+s_{\alpha} s_{\beta} c_{\alpha}-c_{\beta}^{2} c_{\alpha}-c_{\beta} c_{\alpha}^{2}-s_{\alpha} s_{\beta}-c_{\beta}^{2}-c_{\alpha} c_{\beta}-c_{\alpha}^{2}+1\right) \\
& -s_{\alpha} c_{\beta}^{2}+s_{\alpha} c_{\beta} c_{\alpha}+s_{\beta} c_{\beta} c_{\alpha}-s_{\beta} c_{\alpha}^{2}+s_{\alpha} c_{\beta}-s_{\alpha} c_{\alpha}-s_{\beta} c_{\beta}+c_{\alpha} s_{\beta}=0 \\
& \text { for } i=[0,1], j=[-1,1]
\end{aligned}
$$

\subsection{Workspace analysis}

For generating the traces of workspace and singularities, we set limits of springs as $\left[l_{\text {min }}, l_{\text {max }}\right]=[10,30] \mathrm{mm}$ for the 3-SPS-U mechanism and $[7,30] \mathrm{mm}$ for the 4SPS-U mechanism. The lower limit $l_{\text {min }}$ contributes mainly to the boundaries of the aspects centered in $\alpha=\beta=0$ radians. A value of $11 \mathrm{~mm}$ is taken for the parameter $r_{f}$ [5]. With these parameters being set, the workspace is estimated for both the mechanisms under study and a comparison is done based on their maximum tilt limits.

\subsubsection{Workspace for the 3-SPS-U architecture}

The workspace and singularity curves for the 3-SPS-U mechanism are represented in Figure 2a. The blue regions indicate the workspace for the mechanism with the singularity boundaries. The joint limits for the three springs are represented in red lines and they appear superimposed on the plots. Parallel singularities can be observed for the mechanisms at the corners of the blue regions especially at $\alpha=\beta$ $=\pi / 3$ radians. The singularity free workspace for the mechanism is bounded by a triangular region which has to be extracted to determine the minimum limit for the springs. One edge of triangle is extracted at $\alpha=0$ and $\beta=-\pi / 3$ radians. At this position, one or two of the springs reach their minimum position with no singularities. A value of $13.5 \mathrm{~mm}$ is estimated for the lengths $l_{2}$ and $l_{3}$ at this position. This is the minimum limit for which the spring can go to avoid parallel singularities. For deter- 


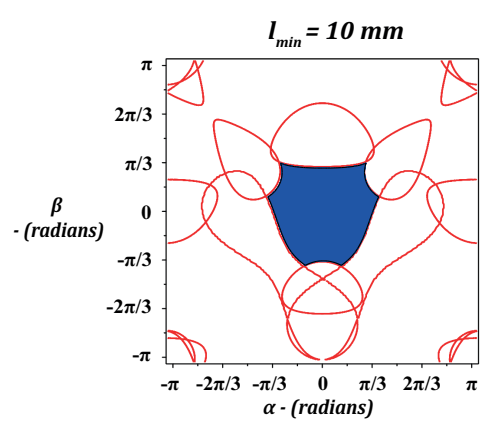

(a)

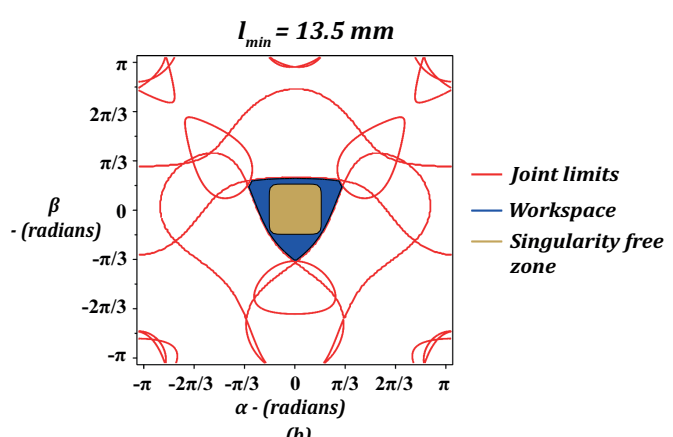

(b)

Fig. 2 Workspace of the 3-SPS-U tensegrity mechanism with singularity boundaries(a) and recalculated singularity free workspace (b)

mining the remaining edges of the triangle, the minimum limits for the other length pairs $\left(l_{1}-l_{2} \& l_{1}-l_{3}\right)$ are fixed as $13.5 \mathrm{~mm}$. The values of $\alpha$ are taken as $\pm \pi / 3$ radians from the workspace obtained in Figure 2a. A value of 0.67 radians is found for $\beta$ at these positions. With the modified lower limit for the springs, the workspace for the 3-SPS-U tensegrity mechanism is recalculated and plotted as shown in Figure $2 \mathrm{~b}$. It could be seen that a singularity free workspace in the form of a Reuleaux triangle is obtained. The minimum square zone within this triangle is bounded by the limits $[\alpha, \beta] \in[-\pi / 6, \pi / 6]$ radians. Thus in order to avoid singularities during operation, the 3-SPS-U mechanism can tilt upto $\pm \pi / 6$ radians.

\subsubsection{Workspace for the 4-S $\underline{P S}-\mathrm{U}$ architecture}

The workspace and singularity surfaces are generated for the 4-SPS-U architecture and it is represented in Figure 3a. In Figure 3a, the joint limits are represented by
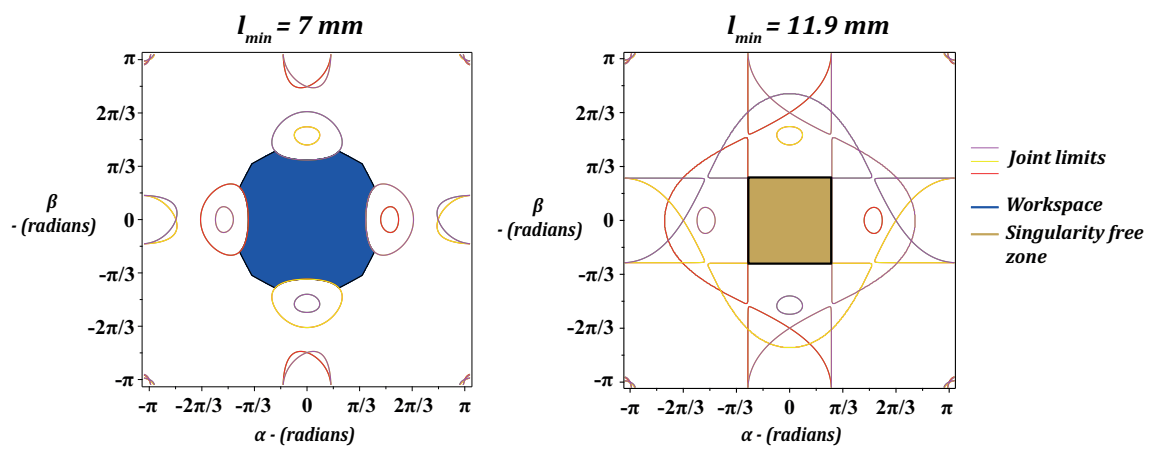

Fig. 3 Workspace of the 4-SPS-U tensegrity mechanism with singularity boundaries(a) and recalculated singularity free workspace (b) 
the colored lines and the workspace is represented by the blue-colored region. Compared to the 3-SPS-U architecture, a wider workspace with singularity regions are observed for the 4-SPS-U mechanism. Based on the analysis, parallel singularities occur when both $\alpha$ and $\beta \in[ \pm \pi / 3, \pm \pi / 3]$ radians. At these boundaries, two of the four legs reaches its minimum length. At $\alpha$ equal to 0 radians, $\beta$ attains maximum tilt values of $\pm \pi / 3$ radians. Using the joint limit Equations $8-9$ and the minimum spring length value at the singularity boundary, the maximum tilt limit with singularity free workspace is estimated for $\alpha, \beta$ at $[0, \pi / 3]$ radians. A tilt limit of $\pm 5 \pi / 18$ radians is obtained and the minimum spring length is estimated to be $11.9 \mathrm{~mm}$. The recalculated workspace is in the form of a square for the 4-SPS-U mechanism and it is represented in Figure $3 b$. The singularity free workspace for this mechanism is superimposed on the square with tilt limits of $\pm 5 \pi / 18$ radians.

\subsection{Discussions}

From the workspace analysis, it could be observed that the 3-SPS-U mechanism generates a higher tilt limit when compared to the 4-SPS-U mechanism. The articulation unit for the bio-inspired robot must be able to overcome pipe bends at $\pi / 2$ radians in a passive mode. With the 3-SPS-U mechanism, it will be difficult to address this problem as there are possibilities that the mechanism might reach singular poses within a narrow tilt limit range. On the other hand, the 4-SPS-U mechanism offers higher tilt limits with a singularity free workspace. The possibilities of reaching singular positions by this mechanism is comparatively less than the 3-SPS-U mechanism. The tilt limit issues for the 3-SPS-U mechanism can be addressed by using stacked modules. A simulation of the 4-SPS-U mechanism coupled with the bio-inspired robot developed at LS2N was performed and it showed that the 4-SPS$\mathrm{U}$ mechanism is capable of overcoming pipe bends at $\pi / 2$ radians.

\section{Conclusions}

In this article, two tensegrity mechanisms were analyzed and compared with respect to the tilt limits obtained from the singularity free workspace. By using the SIROPA library in Maple, the geometric constraints followed by the joint limits and the singularity equations were generated for both the mechanisms. From the resulting singularity free workspaces, it was found that the 4-SPS-U mechanism has higher tilt limits of $\pm 5 \pi / 18$ radians when compared to the 3-SPS-U mechanism which provides $\pm \pi / 6$ radians. The 4-SPS-U mechanism proves to be a better candidate, that can operate within the singularity free workspace in passive modes than the 3-SPS-U mechanism.

In future works, an experiment will be conducted on the tensegrity mechanism in order to correlate with the numerical results. Also, the stability analysis for both 
the mechanisms will be carried out with respect to the Euler angles of the universal joint. This analysis will be done by taking into account the weight of the robot for horizontal and vertical orientations of the pipeline.

Acknowledgements We would like to thank the ANR Avineck project team for the support extended in the initial study of this mechanism.

\section{References}

1. Venkateswaran, S., Chablat, D., Boyer, F.: Numerical and experimental validation of the prototype of a bio-inspired piping inspection robot. Robotics 8(2), 32 (2019)

2. Chen, J., Chen, T., Deng, Z.: Design method of modular units for articulated in-pipe robot inspecting system. In: 2011 Second International Conference on Digital Manufacturing \& Automation, pp. 389-392. IEEE (2011)

3. Hirose, S., Ohno, H., Mitsui, T., Suyama, K.: Design of in-pipe inspection vehicles forpipes. In: Proceedings 1999 IEEE International Conference on Robotics and Automation (Cat. No. 99CH36288C), vol. 3, pp. 2309-2314. IEEE (1999)

4. Brunete, A., Hernando, M., Gambao, E.: Modular multiconfigurable architecture for low diameter pipe inspection microrobots. In: Proceedings of the 2005 IEEE International Conference on Robotics and Automation, pp. 490-495. IEEE (2005)

5. Venkateswaran, S., Furet, M., Chablat, D., Wenger, P.: Design and analysis of a tensegrity mechanism for a bio-inspired robot. In: Proceedings of the ASME 2019 International Design Engineering Technical Conferences \& Computers and Information in Engineering Conference, vol. Volume 5A: 43rd Mechanisms and Robotics Conference (2019). V05AT07A026

6. Shah, S., Saha, S., Dutt, J.: Denavit-hartenberg parameterization of euler angles. Journal of Computational and Nonlinear Dynamics 7(2), 021,006 (2012)

7. Alici, G., Shirinzadeh, B.: Topology optimisation and singularity analysis of a 3-sps parallel manipulator with a passive constraining spherical joint. Mechanism and Machine Theory 39(2), 215-235 (2004)

8. Pan, Y., Chen, Y., Li, L.: Analysis of kinematic dexterity and stiffness performance based on spring's wire-driven 4-sps/u rigid-flexible parallel trunk joint mechanism. International Journal of Structural Integrity (2019)

9. Gosselin, C., Angeles, J.: Singularity analysis of closed-loop kinematic chains. IEEE transactions on robotics and automation 6(3), 281-290 (1990)

10. Jha, R., Chablat, D., Baron, L., Rouillier, F., Moroz, G.: Workspace, joint space and singularities of a family of delta-like robot. Mechanism and Machine Theory 127, 73-95 (2018)

11. Chablat, D., Ottaviano, E., Moroz, G.: A comparative study of 4-cable planar manipulators based on cylindrical algebraic decomposition. In: Proceedings of the ASME 2011 International Design Engineering Technical Conferences \& Computers and Information in Engineering Conference, pp. 1-10 (2011)

12. Chablat, D., Wenger, P.: Working modes and aspects in fully parallel manipulators. In: Proceedings. 1998 IEEE International Conference on Robotics and Automation (Cat. No. 98CH36146), vol. 3, pp. 1964-1969. IEEE (1998) 